\title{
Les temps de la génomique
}

Oléagineux, Corps Gras, Lipides. Volume 9, Numéro 2, 71, Mars - Juin 2002, Editorial

Auteur(s) : Jean-Claude Icart

Résumé : Organisées sur le thème " Génomique et filière oléagineuse ", les Journées Chevreul 2001 de l'AFECG, ont été l'occasion de réunir une des « mailles » du collectif en charge du développement de la génomique végétale et de présenter les principaux résultats de ces recherches concernant les lipides.

\section{ARTICLE}

Organisées sur le thème "Génomique et filière oléagineuse ", les Journées Chevreul 2001 de I'AFECG, ont été l'occasion de réunir une des « mailles » du collectif en charge du développement de la génomique végétale et de présenter les principaux résultats de ces recherches concernant les lipides.

On le sait l'étude du génome des êtres vivants a connu un essor considérable au cours des dix dernières années. Le séquençage des gènes et l'analyse fonctionnelle en cours du génome d'Arabidopsis laissent entrevoir des possibilités d'action importantes en matière d'amélioration des plantes cultivées ${ }^{1}$. Et c'est dans cette perspective d'échanges croisés entre connaissance et applications (sans parler des aspects liés à la concurrence internationale) que la filière des oléagineux est devenue dans le cadre de Biogemma, partenaire du programme Génoplante ${ }^{2}$.

Ces journées offraient ainsi l'opportunité de revisiter ces liens entre recherche et innovation, d'examiner concrètement où en était la prise en compte par ces nouvelles techniques, des demandes des utilisateurs sur les plans agronomique, environnemental et nutritionnel - sujets travaillés dans de multiples instances, mais qui étaient présentés ainsi pour la première fois, du moins pour la filière, à ce niveau public.

Outre les conférences publiées dans le cadre de notre dossier, une partie importante de ces journées fut dans ces conditions, et c'est peu de le dire, occupée par les échanges auxquels elles donnèrent lieu.

La diversité des questions posées par les utilisateurs, la multiplicité des niveaux des réponses scientifiques apportées parfois sous forme de questionnement (" Le rôle des lipides dans les processus de signalisation cellulaire, les enzymes impliquées et les gènes qui les contrôlent sont encore presque inconnus chez les plantes (...) c'est maintenant la recherche des éléments de régulation qui devrait recevoir la priorité des recherches fondamentales ${ }^{3}$ )), la nécessité également pour les tenants d'une vision causale du génome, radicale, d'accepter les médiations d'autres approches (comme le révéla la discussion soulevée par l'idée d'une carte alimentaire personnelle déduite de la carte génomique individuelle) firent ainsi apparaître combien l'élaboration d'un langage commun, intégrant mieux la complexité de ces sujets, est encore une tâche à venir. 
"La diversité des échelles de temps qui caractérisent les différentes étapes de mise au point et de valorisation de ces techniques " sur laquelle André Pouzet attira l'attention dans son discours de conclusion est certainement la cause pratique de cette difficulté. Les différentes logiques de temps qu'exigent la compréhension des différents phénomènes concernés, imposent ainsi plus que jamais, sur un plan scientifique, le temps du débat.

Jean-Claude Icart

Nota

Le dossier "Les nouvelles relations industrie/environnement " à paraître initialement dans le numéro 2 , volume 9 , a été reporté à une livraison ultérieure. Que nos lecteurs veuillent bien nous en excuser.

Avec sa prochaine livraison, volume 9, numéro 4, la revue recouvrera sa pagination habituelle.

${ }^{1}$ OGM et agriculture : options pour l'action publique. Rapport du Commissariat général du Plan. Paris : Documentation française, 2001.

${ }^{2}$ Par l'intermédiaire de Sofiprotéol. Voir l'article de Michel Boucly.

${ }^{3}$ Michel Delseny, page 133. 